\title{
Previsão dos modelos univariados e rede neural da demanda turística do estado de Mato Grosso do Sul
}

\author{
Forecast of univariate models and neural network of tourism demand in the state of Mato Grosso do Sul \\ Predicción de modelos univariantes y red neuronal de demanda turística del estado de Mato Grosso do Sul
}

Bruno Matos Porto

Universidade Federal de Mato Grosso do Sul (UFMS),

DOI: https://doi.org/10.18472/cvt.19n3.2019.1520

Brasil

Redalyc: http://www.redalyc.org/articulo.oa?

bmatosporto@gmail.com

Daniela Althoff Philippi

Universidade Federal de Mato Grosso do Sul (UFMS),

Brasil

daniela.philippi@ufms.br

Vanessa Aline Wagner Leite

Universidade Federal de Mato Grosso do Sul (UFMS),

Brasil

vanessawleite@gmail.com

Recepción: 27 Noviembre 2017

Aprobación: 10 Mayo 2019

\section{Resumo:}

O objetivo deste artigo foi gerar previsões de curto, médio e longo prazos e comparar a precisão dos modelos em cada horizonte de previsão. Para atender o objetivo foram aplicados os modelos univariados e rede neural (NNAR) nos dados da demanda turística do estado de Mato Grosso do Sul (MS). A amostra foi coletada na ferramenta base de dados extrator do Instituto Brasileiro de Turismo (Embratur) referente as chegadas turísticas por todas as vias registradas no MS entre janeiro de 2007 a dezembro de 2017. As previsões dos modelos de previsão ARIMA, Holt-Winters ( $\mathrm{HW}$ ) versões aditiva e multiplicativa e NNAR foram projetadas, por meio da linguagem de programação R, com uso do software R Studio. O procedimento empírico de execução dos scripts de todos os modelos foi disponibilizado. As predições fora da amostra da procura do turismo abrangeram o intervalo de janeiro até dezembro de 2018, sendo então comparadas aos dados reais do mesmo período. As previsões dos modelos foram comparadas no curto, médio e longo prazo mediante os critérios Mea Absolute Percentage Error (MAPE), Mean Absolute Deviation (MAD) e Mean Squared Error (MSD). A rede neural (NNAR) superou os modelos testados em diferentes horizontes de previsão e as medidas de erros mostraram que a NNAR é altamente precisa. Em segundo lugar no ranking de acuracidade destacou-se ARIMA. Os resultados mostraram que as previsões da rede neural auxiliam na tomada de decisão dos planejadores turísticos de MS. Para pesquisas futuras recomenda-se realizar previsões fora da amostra num amplo número de séries temporais.

Palavras-chave: Demanda Turística, Séries Temporais, MS, Tomada de Decisão.

\section{Abstract:}

The purpose of this study was to generate short, medium, and long-term forecasts and compare the accuracy of the models in each forecast horizon. For that purpose, univariate and neural network (NNAR) models were applied to data on tourist demand of the state of Mato Grosso do Sul (MS). The sample was collected from the Brazilian Tourism Institute (Embratur) extractor database tool for tourist arrivals by all routes registered in MS between January 2007 and December 2017. The forecasts of ARIMA, HoltWinters (HW) additive and multiplicative versions, and NNAR were performed through the R programming language, using the R Studio software. The empirical script of execution procedure for all models was made available. The out-of-sample tourism demand forecasts ranged from January to December 2018 and were then compared to actual data for the same period. Model forecasts were compared in the short, medium, and long-terms using the Mea Absolute Percentage Error (MAPE), Mean Absolute Deviation (MAD) and Mean Squared Error (MSD) criteria. The neural network NNAR surpassed the models tested in different forecast horizons and error measurements showed that NNAR is highly accurate. ARIMA stood out in second in the accuracy ranking. The results showed that neural network forecasts help the decision making of tour planners in MS. For future research, it is recommended to make out-of-sample forecasts over a larger number of time series. 
KEYworDs: Tourist Demand, Time series, Mato Grosso do Sul, Decision making.

\section{RESUMEN:}

El propósito de este trabajo fue generar pronósticos a corto, mediano y largo plazos y comparar la precisión de los modelos en cada horizonte de pronóstico. Para cumplir con el objetivo, se aplicaron modelos univariantes y de red neuronal (NNAR) en los datos de la demanda turística del estado de Mato Grosso do Sul (MS). La muestra fue colectada por intermedio de la herramienta de base de datos extractor del Instituto Brasileño de Turismo (Embratur) a las llegadas de turistas por todas las rutas registradas en MS concerniente, entre enero de 2007 y diciembre de 2017. Las previsiones de los modelos de predicción ARIMA, Holt-Winters (HW) versiones aditivas y multiplicativas y NNAR se realizaron a través del lenguaje de programación R, utilizando el software R Studio. Se ha puesto a disposición el procedimiento empírico de ejecución de los scripts para todos los modelos. Los pronósticos no pertenecientes a la muestra de búsqueda del turismo cubrieron el rango de enero a diciembre de 2018 y luego se compararon con los datos reales del mismo período. Las predicciones de los modelos se compararon a corto, mediano y largo plazos, bajo los criterios Mea (MAPE), Mean Absolute Deviation (MAD) y Mean Squared Error (MSD). La red neuronal (NNAR) superó los modelos probados en diferentes horizontes de predicción y las mediciones de errores mostraron que la NNAR es altamente precisa. En segundo lugar en el ranking de precisión se destacó ARIMA. Los resultados mostraron que las predicciones de la red neuronal ayudan a la toma de decisiones de los planificadores turísticos de MS. Para futuras investigaciones, se recomienda hacer pronósticos fuera de la muestra en una gran cantidad de series de tiempo.

Palabras Clave: Demanda turística, Series de tiempo, Mato Grosso do Sul, Toma de decisiones.

\section{INTRODUÇÃO}

As predições automáticas de um amplo número de séries cronológicas univariadas são frequentemente indispensáveis nos negócios (HYNDMAN; KHANDAKAR, 2008). A atividade turística necessita de um planejamento de previsão de demanda turística (CHU, 1998; SERRA; TAVARES; SANTOS, 2005). Neste enfoque é consenso que planejamento relaciona-se com as decisões que serão tomadas e afetará o futuro (GOH; LAW, 2002).

As previsões em curto prazo são relevantes para vários objetivos de gestão como bem explicados por Gunter e Önder (2015, p. 123): "previsão de curto prazo da previsão da demanda nos próximos dois ou três meses pode ajudar o destino a ter mais flexibilidade operacional, por exemplo, em termos de número de ônibus do aeroporto para o centro da cidade". Diante disso, os serviços turísticos necessitam de um planejamento preditivo que é obtido via predição acurada da demanda turística (GOH; LAW; MOK, 2008). Outro aspecto significativo de dados da demanda turística é a natureza da sazonalidade que deve ser identificada antes da seleção do modelo de previsão ideal que a considere (GOH; LAW, 2002).

O estudo é importante devido aos impactos do fenômeno turístico na economia do Brasil e sua parte considerável no desenvolvimento econômico e social, por meio de geração de empregos diretos, indiretos e renda (CASANOVA et al., 2012), tendo em vista que a indústria do turismo no Brasil, com base nos dados disponíveis pela Embratur, representa 9\% do PIB, assim gerou direta e indiretamente mais de 7,5 milhões de empregos no Brasil, além disso, mantém milhões de micro e pequenas empresas de muitos segmentos da economia (EMBRATUR, 2017a).

A previsão de demanda do turismo é um assunto significativo para os praticantes e acadêmicos (SONG; LI, 2008; KIM et al., 2011; GUNTER; ÖNDER, 2015). A predição de demanda turística desempenha um papel fundamental no planejamento turístico uma vez que os projetos de investimento no turismo que utilizam altos recursos financeiros precisam de uma previsão precisa do que irá ocorrer futuramente com a demanda (CHO, 2003). É amplamente concordado que as regiões específicas requerem previsões precisas da procura do turismo, mas existem poucas pesquisas neste sentido devido à falta de informação estatística (CLAVERIA; TORRA, 2014).

A pesquisa de Chu (1998) aplicou os modelos ARIMA sazonal e não sazonal, Holt-Winters nos dados de chegada de turistas nos países da Ásia-Pacífico. Os resultados revelaram que a previsão no curto ou médio prazo de ARIMA sazonal e não sazonal foram os mais acurados dos modelos testados, jugado pelo critério 
MAPE, assim, testou-se a acuracidade dos modelos ARIMA sazonal e não sazonal, Holt-Winters, por meio de MAPE e receberam um percentual abaixo de $10 \%$.

No estudo de Goh e Law (2002) aplicaram-se os modelos SARIMA e MARIMA, com intervenções nas dez séries temporais de chegadas mensais para Hong Kong de dez países de origem e, para avaliar os desempenhos das previsões, utilizaram-se os índices de desempenho MAD, RMSE, RMSPE, MSE, MAPE e Theil's U. Destacou-se o modelo preditivo MARINA, no entanto depende do mercado de origem, pois em alguns mercados o desempenho individual do modelo SARIMA supera as previsões de MARINA.

Numa revisão de cento e vinte e uma publicações sobre previsão da demanda turística e modelagem de 2000 até 2007, na Escola de Administração de Hotelaria e Turismo da Universidade Politécnica de Hong Kong, concluiu-se que não há modelo de previsão que em diferentes situações tenha um desempenho de previsão melhor com relação aos demais modelos na literatura especializada de previsão da demanda (SONG; LI, 2008).

$\mathrm{Na}$ procura do turismo de Paris (França) compararam-se modelos univariados (ARIMA, ES e Naive 1) e multivariados (VAR clássico e bayesiano, TVP, EC-ADLM) de previsão com a finalidade de avaliar a acuracidade dos sete modelos mediante as medidas de erro RMSE e MAE. Os resultados evidenciaram que todos os modelos testados tiveram melhor desempenho preditivo do que o modelo Naive 1, em vários horizontes de predição e mercados de origem (GUNTER; ÖNDER, 2015).

$\mathrm{Na}$ previsão em curto, médio e longo prazos dos dados de chegadas turísticas em países da Europa, através da aplicação dos modelos ARIMA, ETS, NN, TBATS, ARFIMA, MA, WMA, SSA-R e SSA-V. E a comparação da acurácia destes modelos pela medida de discrepância RMSE, DC e DM. Constatou-se que em diferentes horizontes de previsão e mercados pesquisados, não se identificou um único modelo que tivesse melhor desempenho do que todos os diversos modelos testados em todas as circunstâncias (HASSANI et al., 2017). A partir desses achados, a pesquisa objetivou gerar previsões de curto, médio e longo prazos e comparar a precisão dos modelos em cada horizonte de previsão.

Portanto, o problema de pesquisa do artigo que ora se apresenta foi como as previsões da demanda turística, por meio dos modelos, podem contribuir no processo de tomada de decisão dos planejadores turísticos de Mato Grosso do Sul? Foi realizada a aplicação dos modelos univariados (ARIMA automático, Holt-Winters versões aditiva e multiplicativa) e rede neural com intervenção. No contexto da demanda turística de MS em uma única série temporal de chegadas turísticas internacionais.

\section{BASE TEÓRICA}

A seguir apresentam-se, com base na literatura consultada, demanda turística, Holt-Winters (HW) versões aditiva e multiplicativa, ARIMA, rede neural e medida de precisão.

\subsection{Demanda turística}

Nas últimas décadas, houve um crescimento significativo da procura do turismo no mundo (GOH; LAW, 2002; SONG; LI, 2008; HASSANI et al., 2017). Além disso, os serviços turísticos têm uma natureza perecível, o que estabelece a necessidade de previsão precisa da demanda do turismo (GOH; LAW; MOK, 2008; GUNTER; ÖNDER, 2015).

A predição da demanda turística de uma cidade é fundamental para a estratégia de planejamento do ponto de vista do turismo que proporciona expansão dos serviços turísticos, tanto em investimento de recursos humanos (treinamento) como estrutura física, para atender as necessidades das pessoas (CASANOVA et al., 2012). Neste sentido, Claveria e Torra, (2014, p. 220) afirmam que "as previsões precisas do volume do 
turismo desempenham um papel importante no planejamento do turismo, pois permitem que os destinos prevejam necessidades de desenvolvimento de infraestrutura”.

Alguns motivos para prever uma futura demanda turística são apontados por Gunter \& Önder (2015, p. 123):

O quarto do hotel que não é vendido, amanhã será uma perda de receita. Um destino também precisa saber sobre quantos turistas são esperados para que a quantidade de voos para o destino pode ser ajustado, novos hotéis podem ser construídos e funcionários adicionais podem ser contratados.

\subsection{Holt-Winters $(\mathrm{HW})$}

O modelo foi criado em 1960 por Winters; este modelo de previsão é uma extensão do modelo de Holt, assim apresenta um parâmetro a mais e equação complementar para a sazonalidade (MAKRIDAKIS, 1976). Dentre suas principais vantagens são a sua simples utilização (CHATFIELD, 1978; CHATFIELD; YAR, 1988; SILVER, 2000); o seu bom desempenho, de modo geral e na prática (CHATFIELD; YAR, 1988); o baixo custo de operação (NEWBOLD; GRANGER, 1974; SERRA; TAVARES; SANTOS, 2005) e útil para prever como o modelo complexo de Box-Jenkins (SILVER, 2000).

O modelo de série temporal HW tem muita utilidade para os decisores e planejadores estratégicos que visam um modelo econômico e relativamente preciso com a finalidade de amplo horizonte de previsão e significativo número de séries de tempos (BRANDON; JARRETT; KHUMAWALA, 1987).

De acordo com Chatfield (1978), há dois tipos de modelo sazonal: uma versão aditiva, para a qual se supõe que os efeitos sazonais são de tamanho constante; e a versão multiplicativa, para a qual se supõe que os efeitos sazonais são proporcionais em tamanho ao nível médio dessazonalizado local. $\mathrm{O}$ modelo HoltWinters baseia-se em quatro equações apresentadas a seguir: a primeira, o componente nível (1), em seguida, componente tendência (2) e, então, o componente sazonal (3) e, por fim, as previsões do tempo (4). As equações das versões multiplicativa e aditiva são exibidas na sequência.

$$
\begin{gathered}
\mathrm{L}_{\mathrm{t}}=\alpha\left(\frac{\mathrm{x}_{\mathrm{t}}}{\mathrm{S}_{\mathrm{t}-\mathrm{m}}}\right)+(1-\alpha)\left(\mathrm{L}_{\mathrm{t}-1}+\mathrm{T}_{\mathrm{t}-1}\right) \\
\mathrm{T}_{\mathrm{t}}=\beta\left(\mathrm{L}_{\mathrm{t}}-\mathrm{L}_{\mathrm{t}-1}\right)+(1-\beta) \mathrm{T}_{\mathrm{t}-1} \\
\mathrm{~S}_{\mathrm{t}}=\gamma\left(\frac{\mathrm{x}_{\mathrm{t}}}{\mathrm{L}_{\mathrm{t}}}\right)+(1-\gamma) \mathrm{S}_{\mathrm{t}-\mathrm{m}} \\
\mathrm{F}_{\mathrm{t}+\mathrm{n}}=\left(\mathrm{L}_{\mathrm{t}}+\mathrm{nT}_{\mathrm{t}}\right) \mathrm{S}_{\mathrm{t}-\mathrm{m}+\mathrm{n}}
\end{gathered}
$$


Fonte: Adaptado de Chatfield (1978)

$\mathrm{Na}$ versão aditiva, são as equações:

$$
\begin{gathered}
\mathrm{L}_{\mathrm{t}}=\alpha\left(\mathrm{x}_{\mathrm{t}}-\mathrm{S}_{\mathrm{t}-\mathrm{m}}\right)+(1-\alpha)\left(\mathrm{L}_{\mathrm{t}-1}+\mathrm{T}_{\mathrm{t}-1}\right) \\
\mathrm{T}_{\mathrm{t}}=\beta\left(\mathrm{L}_{\mathrm{t}}-\mathrm{L}_{\mathrm{t}-1}\right)+(1-\beta) \mathrm{T}_{\mathrm{t}-1} \\
\mathrm{~S}_{\mathrm{t}}=\gamma\left(\mathrm{x}_{\mathrm{t}}-\mathrm{L}_{\mathrm{t}}\right)+(1-\gamma) \mathrm{S}_{\mathrm{t}-\mathrm{m}} \\
\mathrm{F}_{\mathrm{t}+\mathrm{n}}=\mathrm{L}_{\mathrm{t}}+\mathrm{nT}_{\mathrm{t}}+\mathrm{S}_{\mathrm{t}-\mathrm{m}+\mathrm{n}}
\end{gathered}
$$

Fonte: Adaptado de Chatfield (1978)

Onde: - estimativa do grau médio dessazonalizada no período $t$; - fator sazonal estimado para o tempo t; - prazo de tendência estimado para o tempo $t ; \mathrm{m}$ - número de considerações em ciclo sazonal; - predições de período $\mathrm{n}$ em diante; $\mathrm{n}-1,2, \ldots$; - número observado e - constantes de ponderação de nível, tendência e sazonalidade, nesta ordem (CHATFIELD, 1978).

\subsection{Modelos Auto-Regressivos Integrados de Médias Móveis (ARIMA)}

As séries cronológicas são formadas de valores observados em vários períodos ordenados e subsequentes ( STEVENSON, 1981; KAZMIER, 1982). Dentre os modelos utilizados para analisar uma série de tempo univariada tem-se o ARIMA. Proposto por Box-Jenkins em 1976, o modelo usa uma combinação de três componentes: autorregressivo (AR), filtro de integração (I) e médias móveis (MA) (BUNDCHEN; WERNER, 2016).

O modelo ARIMA utilizado nesta pesquisa é uma versão automática denominada auto.arima. Onde o valor de d é escolhido com base em repetidos testes de raiz de unidade KPSS, em seguida são selecionados os valores de p e q minimizando o Critério de Informação Akaike (AIC) para determinar os valores.

$$
\phi(\mathrm{B})\left(1-\mathrm{B}^{d}\right) \mathcal{Y}_{t}=c+\theta(\mathrm{B}) \varepsilon_{t}
$$


Onde, é um processo de ruído branco com zero médio e variância , é o operador de mudança de turno, e e são polinômios da ordem p e q, respectivamente. Para garantir causalidade e invertibilidade, presume-se que e não têm raízes para (BROCKWELL; DAVIS, 1991). Se, existe um polinômio implícito de ordem d na função de previsão (HYNDMAN; KHANDAKAR, 2008). O modelo de previsão automático ARIMA seleciona a ordem de p, q e d, por meio do critério de Informação conforme o AIC:

$$
\mathrm{AIC}=-2 \log (L)+2(p+q+P+Q+k)
$$

Em que $=1$ se e 0 de outra forma, e é a probabilidade máxima do modelo fixado para dados diferidos . A probabilidade de o modelo completo para não é realmente definida e, portanto, o valor da AIC para diferentes níveis de difusão não é comparável (HYNDMAN; KHANDAKAR, 2008).

Assim, o algoritmo Hyndman-Khandakar seleciona o modelo que apresenta o menor valor AIC de: ARIMA (2, d, 2), ARIMA (0, d, 0), ARIMA $(1, d, 0)$ e ARIMA $(0, d, 1)$ dentre os quatros modelos (HASSANI et al., 2017). Para os leitores mais interessados nos detalhes de funcionamento da modelagem ARIMA basta ver a seção 8.7 em (HYNDMAN; ATHANASOPOULOS, 2018a).

\subsection{Rede neural}

A procura do turismo é uma área especialmente importante na análise de dados para predição e acurácia do desempenho de previsão de redes neurais em comparação aos modelos de séries temporais (CLAVERIA; TORRA, 2014).

A rede neural de Feed-forward constitui-se em camadas: uma de entrada, uma de saída e, de modo geral uma ou mais camadas escondidas e a capacidade de aprendizagem em reconhecer padrões desta rede neural possibilita ser utilizada como um modelo de previsão aplicado nos negócios (LAW, 2000). As entradas constituem a camada inferior e nas saídas formam a camada superior é possível ter camadas ocultas (HYNDMAN; ATHANASOPOULOS, 2018b).

Diante da flexibilidade das redes neurais, elas não têm um processo sistêmico para criação de modelos, logo a obtenção de um modelo de rede neural robusto ocorre mediante a escolha de um grande número de parâmetros que são obtidos experimentalmente, por meio, de tentativa e erro (PALMER; JOSÉ MONTAÑO; SESÉ, 2006). Este método foi utilizado neste estudo para selecionar a rede neural especificada aqui. Os detalhes sobre o funcionamento do modelo nnetar pode ser visto na seção 11.3, em (HYNDMAN; ATHANASOPOULOS, 2018b). A figura 1 apresenta a arquitetura geral de uma rede do tipo Feed-forward.

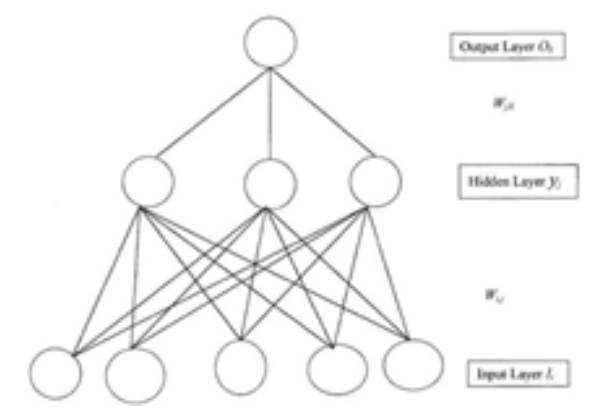

Figura 1 - Simplificação rede neural com três camadas Fonte: Elaborado por Law (2000) 


\section{MEDIDA DE PRECISÃO}

A partir da explicação que devesse julgar a adaptação de qualquer medida de erro utilizada pelo resultado gerado da precisão das previsóes fora da amostra do modelo de previsão, assim uma medida de erro para ser selecionada precisa aliar-se a finalidade da predição e os objetivos dos decisores (MAKRIDAKIS, 1993). Neste contexto, numa pesquisa bibliográfica sobre previsão de demanda turística de 2005 até 2015, com um total de 129 artigos, (BUNDCHEN, 2016), constatou-se que Mea Absolute Percentage Error (MAPE) é o critério mais usado na predição da procura do turismo.

O MAPE fornece os resultados em porcentagem que facilita a interpretação pelos decisores, bem como gera um indicativo qualitativo da precisão do modelo de previsão testado (FLORES, 1986; MAKRIDAKIS, 1993). O Mean Absolute Deviation (MAD) mesura a acuracidade global e gera um indicativo da disseminação global em que todos os erros são dados pesos iguais (LAW, 2000). A medida é mesurada nas mesmas unidades dos dados históricos, além disso, é fácil para demonstrar aos tomadores de decisão por conta da facilidade de cálculo (FLORES, 1986). Neste sentido, MAD constitui-se numa das primeiras medidas de erro, sendo singela de calcular e simples de compreender (BONETT; SEIER, 2003).

O Mean Squared Error (MSD) é uma medida comumente usada para entender a adaptação de um método de previsão em relação aos dados e medir o desempenho das predições geradas, o critério também é fácil de calcular e de modo geral um bom critério para medir o desempenho de previsões da única série de tempo (CHATFIELD, 1988).

Este critério é comumente utilizado para comparar métodos de previsão, além de verificar o erro quadrático, se diferencia de outras medidas de precisão, pois demonstra qual modelo minimiza grandes erros uma vez que mostra estes erros mais do que outros critérios (THOMPSON, 1990).

$$
\begin{gathered}
M A D=\frac{\sum|R-D|}{n} \\
M S D=\frac{\sum(R-D)}{n-1} \\
\cdots \\
M A P E=\frac{\sum|R-D / R|}{n} \cdot 100,(R \neq 0)
\end{gathered}
$$

Fonte: Adaptado de (MIN, 2008)

Sendo, $\mathrm{R}$ = valor real de demanda; $\mathrm{D}$ = previsão; $\mathrm{n}$ = número de observações.

Medidas de erro são métodos de gerar informações sobre a precisão de certo modelo de previsão para predizer os dados reais, tanto para modelos que são ajustadas aos dados quanto para previsóes de períodos, fora da amostra (MAKRIDAKIS, 1993).

\section{PROCEDIMENTOS METODOLÓGICO}

Nesta seção descreve-se a área de estudo, coleta de dados, pacote usado da linguagem de programação R, no $\mathrm{R}$ studio e o procedimento prático de execução dos scripts de todos os modelos. 


\section{1 Área de estudo}

O Estado do Mato Grosso do Sul (Figura 2), localizado no centro-oeste brasileiro, possui cerca de $357.145,531 \mathrm{Km}^{2}$ e conta com uma população estimada em mais de 2,5 milhões de habitantes (IBGE, 2016). Situado em região transfronteiriça com Paraguai e Bolívia a Oeste, faz divisa com os Estados brasileiros Paraná, São Paulo, Minas Gerais e Goiás a Leste e o Estado de Mato Grosso ao Norte.

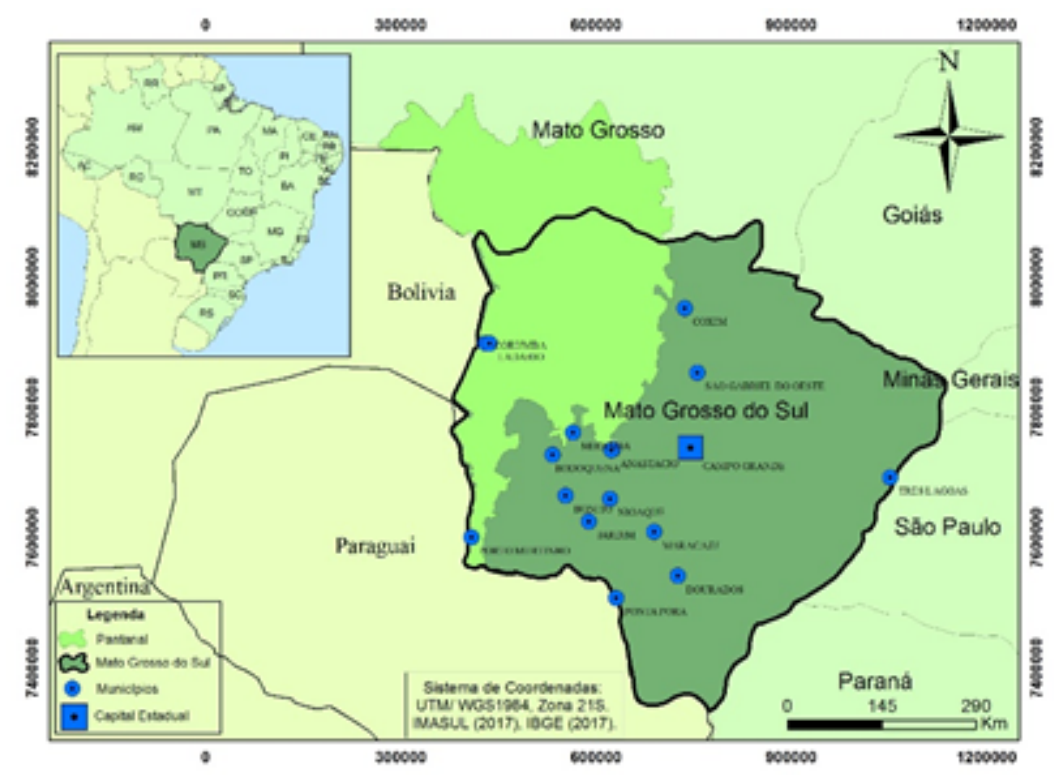

Figura 2 - Localização do Estado de Mato Grosso do Sul, Brasil. Fonte: Elaborado pelos autores através do software ARCGIS (2012)

O estado encontra-se em parte inserido em uma importante unidade geológica como a Bacia Sedimentar do Pantanal, que lhe confere potencialidades turísticas manifestadas nos municípios de Corumbá, Miranda e Aquidauana correspondendo ao Pantanal e nos municípios de Bodoquena, Bonito e Jardim pela relevância de seu ambiente cárstico (MATO GROSSO DO SUL, 2017) localizado na zona de transição da borda do Pantanal sul-matogrossense. Essa unidade representa também um importante cenário hidrográfico, além da riqueza em sua biodiversidade e da beleza paisagística.

O Pantanal, sendo a maior planície alagável do mundo e patrimônio da humanidade segundo a Organização das Nações Unidas para a Educação, a Ciência e a Cultura (UNESCO) (MATO GROSSO DO SUL, 2017), abrange entre suas atividades turísticas o ecoturismo, buscando realizar suas atividades de maneira sustentável preservando e conservando as belezas naturais. São desenvolvidos o turismo cultural relacionado a festas e eventos culturais do estado e o turismo de pesca esportiva, em virtude da riqueza fluvial do rio Paraguai e seus afluentes. Suas atividades contam com pousadas pantaneiras que oferecem pacotes para contemplação da fauna e flora, passeios de chalanas pelos rios da bacia hidrográfica do rio Paraguai, observação de aves típicas da região, safaris e voos panorâmicos (ROTTA; LUNA; WEIS, 2006).

Adjacente ao Pantanal, devido ao seu relevo cárstico e rochas carbonáticas, a região de Bodoquena, Bonito e Jardim possui peculiaridades turísticas com seus "rios de águas cristalinas que abrigam exuberante fauna ictiológica; cachoeiras, fauna terrestre, flora; grutas e ainda pelo contraste da planície com a Serra da Bodoquena" (BARBOSA; ZAMBONI, 2000, p. 06) apresentando "inúmeras cavernas, dolinas, ressurgências e sumidouros, dentre outras feições, além de promover uma extrema limpidez dos cursos d'água que nela têm suas nascentes" (MATO GROSSO DO SUL, 2010, p. 15). 


\subsection{Coleta de dados}

Os dados foram coletados a partir da ferramenta base de dados extrator que de acordo com o (EMBRATUR, 2017b) é:

O extrator de dados sobre a Chegada de turistas internacionais não residentes ao Brasil é uma ferramenta eletrônica interativa que representa importante incremento na disseminação de informações sobre o setor turismo relativas Brasil, unidades da federação, regiões.

Além disso, serve para "A série disponibilizada permite o acompanhamento da evolução do fluxo receptivo internacional de turistas, e dá ao usuário a flexibilidade, rapidez, precisão e consistência de informações que a análise gerencial de informações requer" (EMBRATUR, 2017c). Um detalhe importante sobre a coleta e a ferramenta é que "O extrator de dados é uma customização do Saiku, software de código aberto para análise de dados desenvolvida pela Meteorite BI” (EMBRATUR, 2017d).

Tanto a ferramenta como as informações são disponibilizadas pelo Instituto Brasileiro de Turismo (Embratur) na web site da empresa: http://basededados.turismo.gov.br/. Portanto mediante a base de dados a coleta foi referente às chegadas turísticas entre os períodos de janeiro de 2007 a dezembro de 2017, que seguiu os seguintes procedimentos: Foi selecionado na base em conteúdo: chegadas turísticas, na opção dimensões: mês, mês, todos os meses, via de acesso, todas, nível geográfico, unidade da federação, ano, todos os anos, ano, nesta ordem. Para exibir os dados por região com anos nas colunas e meses nas linhas dessa forma foi mais fácil de organizar a amostra com o objetivo de importar para o R. Os dados utilizados encontramse na quadro 1 :

Quadro 1 - Chegadas turísticas internacionais por todas as vias para Mato Grosso do Sul de janeiro de 2007 a dezembro de 2017

\begin{tabular}{|l|c|c|c|c|c|c|c|c|c|c|c|}
\hline Mês/Ano & $\mathbf{2 0 0 7}$ & $\mathbf{2 0 0 8}$ & $\mathbf{2 0 0 9}$ & $\mathbf{2 0 1 0}$ & $\mathbf{2 0 1 1}$ & $\mathbf{2 0 1 2}$ & $\mathbf{2 0 1 3}$ & $\mathbf{2 0 1 4}$ & $\mathbf{2 0 1 5}$ & $\mathbf{2 0 1 6}$ & $\mathbf{2 0 1 7}$ \\
\hline Janeiro & 6.888 & 6.715 & 19.747 & 21.260 & 5.477 & 7.314 & 6.031 & 8.454 & 7.542 & 12.544 & 11.943 \\
\hline Fevereiro & 5.412 & 6.073 & 13.896 & 15.066 & 4.469 & 3.205 & 4.165 & 5.129 & 4.994 & 6.687 & 6.886 \\
\hline Março & 4.583 & 6.252 & 13.527 & 14.540 & 2.890 & 4.314 & 4.129 & 3.909 & 4.400 & 6.011 & 5.060 \\
\hline Abril & 3.406 & 2.922 & 3.821 & 4.167 & 4.045 & 2.993 & 3.144 & 4.302 & 4.533 & 5.003 & 5.545 \\
\hline Maio & 3.716 & 3.243 & 1.162 & 2.952 & 3.072 & 1.897 & 2.290 & 4.085 & 3.697 & 4.262 & 4.789 \\
\hline Junho & 3.080 & 2.675 & 955 & 3.244 & 2.142 & 4.196 & 2.269 & 6.905 & 3.250 & 4.278 & 4.131 \\
\hline Julho & 5.957 & 3.462 & 2.035 & 2.221 & 2.528 & 4.072 & 3.694 & 4.512 & 4.941 & 6.212 & 6.569 \\
\hline Agosto & 5.946 & 3.702 & 1.401 & 1.538 & 1.402 & 2.919 & 2.817 & 3.867 & 3.508 & 5.398 & 6.110 \\
\hline Setembro & 3.542 & 3.070 & 964 & 1.058 & 1.467 & 2.545 & 1.230 & 3.488 & 1.636 & 4.973 & 6.233 \\
\hline Outubro & 3.660 & 2.746 & 361 & 652 & 2.866 & 2.309 & 2.745 & 3.334 & 4.192 & 4.946 & 5.513 \\
\hline Novembro & 3.809 & 3.431 & 269 & 876 & 3.241 & 1.732 & 2.945 & 3.732 & 4.225 & 5.716 & 5.651 \\
\hline Dezembro & 5.210 & 5.217 & 257 & 566 & 5.501 & 6.395 & 6.064 & 10.282 & 9.683 & 10.998 & 11.840 \\
\hline
\end{tabular}

Fonte: Adaptada pelos autores a partir de (EMBRATUR, 2017e)

Foi aplicada a estatística descritiva na figura 2, da amostra, para resumir as estatísticas com informações básicas como média, desvio padrão e mediana. 


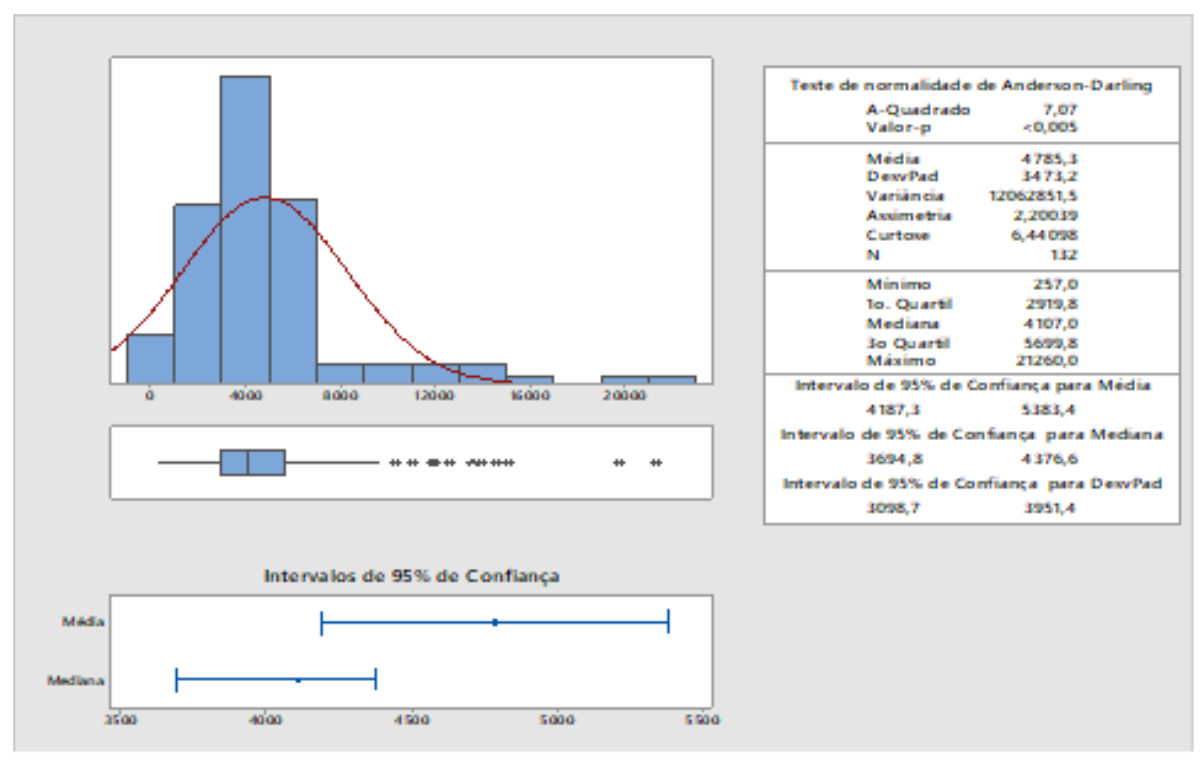

Figura 3 - Estatísticas descritivas e distribuição da amostra de chegadas turísticas Fonte: Elaborado pelo autor a partir do MINITAB 2018

Foram calculadas as estatísticas descritivas das amostras por meio do software Minitab 2018, versão 18.1. Com base na figura 2, 95\% da amostra estão entre 3694 e 5383 chegadas, conforme a distribuição. A amostra também apresenta forte componente sazonal principalmente nos meses de janeiro, fevereiro e dezembro.

Os outliers do gráfico boxplot são as observações da série nos respectivos meses de janeiro até dezembro, neste caso essas observações não podem ser retiradas da amostra, em virtude da proposta da pesquisa ser proceder previsões de valores futuros da série, assim séries temporais com observações faltantes podem prejudicar o desempenho do modelo de previsão. A estatística descritiva elaborada teve como objetivo proporcionar um resumo geral da amostra.

Após a coleta da amostra para avaliar a sua própria normalidade foi aplicada a estatística Shapiro-Wilk Test (SWT) em R usando a função shapiro.test para detectar a distribuição normal na amostra. O ShapiroWilk Test (SWT) é um poderoso e competitivo teste para a normalidade (LEE; QIAN; SHAO, 2014). Realizado o shapiro.test e analisados os resultados apresentados na tabela 1 , verifica-se que a amostra possui uma distribuição normal.

Tabela 1 - Resultado Shapiro-Wilk Test (SWT)

\begin{tabular}{lcc}
\hline Shapiro-Wilk & W & p-value \\
\hline \hline & 0.79 & $3.437 \mathrm{e}-12$ \\
\hline
\end{tabular}

Elaborada pelos autores

\subsection{Pacote em R studio}

Para fazer as previsões e comparar a capacidade preditiva dos modelos ARIMA automático, HW versões aditiva, multiplicativa e rede neural do tipo feed-forward, foi adotado o apoio da linguagem de programação R. Procederam-se as análises de previsão e acuracidade das previsões no $\mathrm{R}$ studio, que é uma extensão do ambiente $\mathrm{R}$, mediante o pacote forecast do R. Os cálculos das medidas de erro MAD, MAPE e MSD também foram executados com o pacote forecast no R studio. (HYNDMAN et al., 2018). 
As funções em $\mathrm{R}$ utilizadas para prever os valores no curto, médio e longo prazos são auto.arima, forecast.HoltWinters e nnetar, (HYNDMAN; KHANDAKAR, 2008; HYNDMAN, 2017). Já para comparar a acuracidade dos modelos de previsão foi usada a função accuracy.

$\mathrm{O}$ modelo de rede neural usado nesta pesquisa pertence ao pacote do $\mathrm{R}$, denominado forecast e o modelo de previsão presente no manual do pacote chama-se nnetar este modelo funciona de forma semelhante aos neurônios do cérebro, assim a arquitetura da rede neural é composta por camadas.

Atende-se as orientações de (HYNDMAN; ATHANASOPOULOS, 2018b), que afirmam "O número de camadas ocultas e o número de nós em cada camada oculta devem ser previamente especificados”. Assim, define-se $\mathrm{p}=4, \mathrm{P}=6$, size $=4$, lambda $=2$, decay $=0.40$. Onde: $\mathrm{p}$ - Dimensão de incorporação para séries temporais não sazonais; $\mathrm{P}$ - Número de atrasos sazonais utilizados como insumos; size - Número de nós na camada oculta; lambda - Parâmetro de transformação Box-Cox; decay - parâmetro de decaimento (HYNDMAN et al., 2018).

Apresentaram-se os pacotes e funções necessárias para aplicar os modelos de previsão, neste procedimento prático, para os pesquisadores e planejadores turísticos que tiverem a necessidade de executar a aplicação dos modelos utilizados na pesquisa, mas em outras amostras. Foi disponibilizada a execução dos scripts com todas as linguagens necessárias para rodar os modelos e as instruções em cada etapa. Nos quadros 2 e 3, demonstrase os procedimentos práticos para aplicação dos modelos de previsão. 
Quadro 2 - Aplicação prática em R do modelo ARIMA

\begin{tabular}{|c|}
\hline $\begin{array}{l}\text { ARIMA para séries temporais univariadas } \\
\text { Emseridos negrito são as linguagens que devem ser alteradas conforme os dados }\end{array}$ \\
\hline te \\
\hline \\
\hline $\begin{array}{l}\text { strams,start }=c(2007,1) \text {, end }=c(2017,12) \text {, frequency=12) \#Cria a } \\
\text { a amostra para fazer as previsões (nessa etapa define-se qual o } \\
\text { ral para previsões fora da amostra) }\end{array}$ \\
\hline ) \\
\hline $\begin{array}{l}\text {,frequency }=12 \text { ) } \\
\text { dos modelos }\end{array}$ \\
\hline lab = "Demanda turística de MS") \#Plotar \\
\hline $\begin{array}{l}\text { egend("topleft",c("Demanda"), cex=0.8, col=c("black"), lty=1, pch=1) \#inserir } \\
\text { egenda no gráfico }\end{array}$ \\
\hline a ponto circula \\
\hline$\#$ Roda c \\
\hline as previsões numa \\
\hline (2) \\
\hline $\begin{array}{l}\text { "black", "blue", pch=1) \# Não fechar a janela do gráfic } \\
\text { a janela a legenda não é inserida }\end{array}$ \\
\hline $\begin{array}{l}\text { legend("topleft", c("Amostra","Previsões de } \quad \text { ARIMA"), cex=0.8, } \\
\text { col=c("black","blue"), lty=1) \# não fechar a janela do gráfico por conta do erro o } R \text { não } \\
\text { inseri a legenda se a janela for fechada }\end{array}$ \\
\hline res de previsão em números \\
\hline 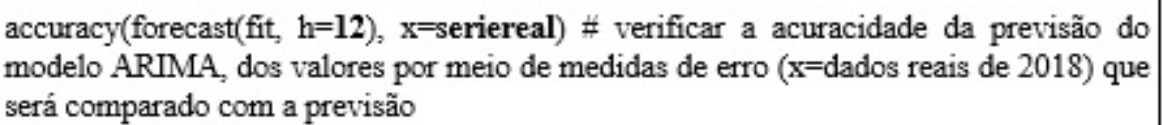 \\
\hline
\end{tabular}

Elaborado pelos autores com base em (HYNDMAN; KHANDAKAR, 2008)

Quadro 3 - Aplicação prática em R da rede neural do tipo Feed-forward

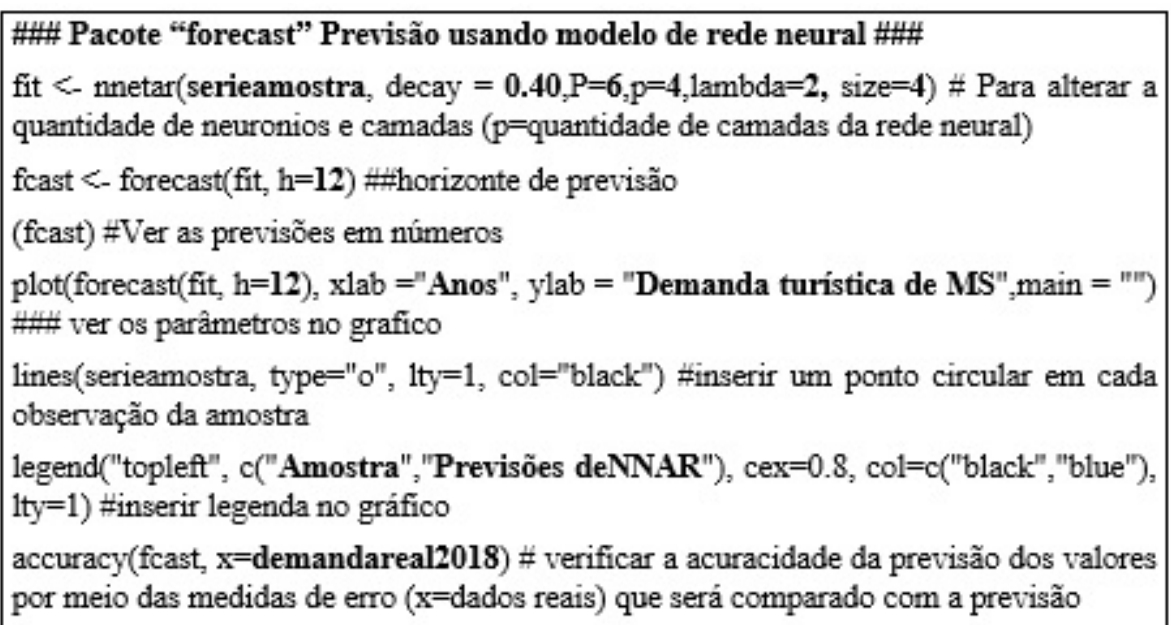


Elaborado pelos autores com base em (HYNDMAN; KHANDAKAR, 2008)

Quadro 4 - Aplicação prática em R dos modelos Holt-Winters versões aditiva e multiplicativa

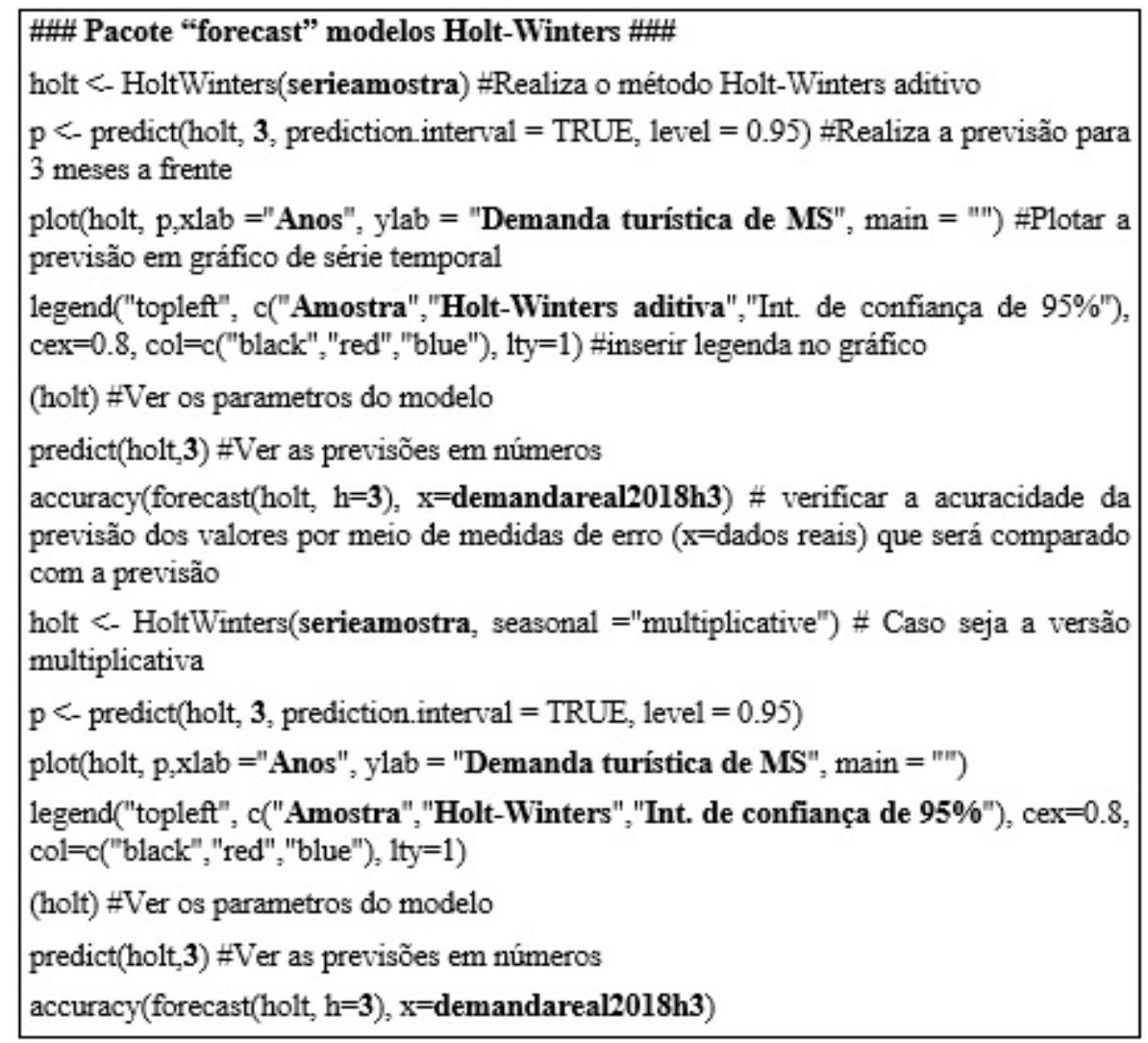

Elaborado pelos autores com base em (HYNDMAN; KHANDAKAR, 2008)

Esses recursos computacionais são de livre acesso e atualmente disponíveis para planejadores turísticos e acadêmicos. No momento em que esses dados foram coletados não estavam disponíveis pela Embratur as observações mais atuais de 2019, por essa razão determinou-se o último ano, 2018, como o período de previsão.

\section{RESULTADOS DA PESQUISA}

princípio plotou-se um gráfico de série temporal mediante os dados coletados de chegadas turísticas e atendem-se as orientações de (HYNDMAN; ATHANASOPOULOS, 2018c), que recomendam inicialmente mostrar os dados em gráfico, construiu-se o gráfico de série temporal representado na figura 3. 


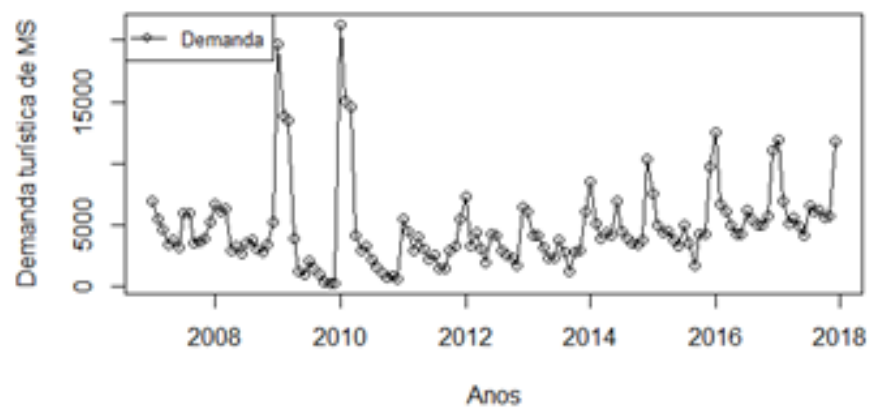

Figura 4 - Chegadas turísticas internacionais por todas as vias Fonte: Elaborado pelos autores por meio do R com base em dados da (EMBRATUR, 2017e)

Assim, contemplando o período entre janeiro de 2007 até dezembro de 2017, verificou-se o comportamento das observações (figura 3).

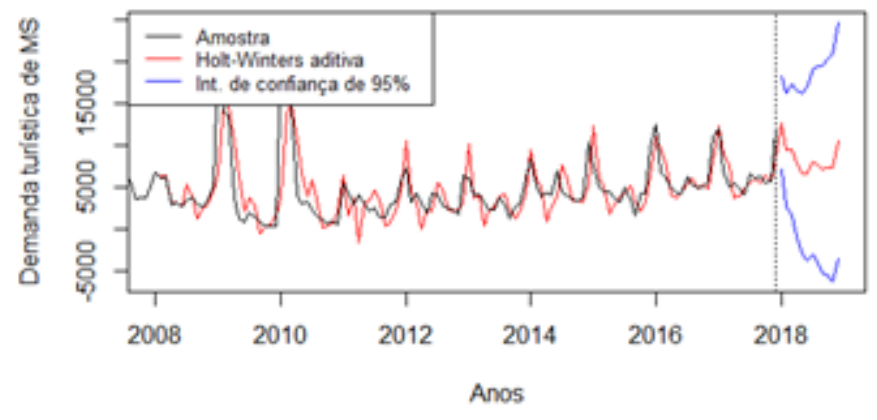

Figura 5 - Previsões de Holt-Winters versão aditiva de janeiro até dezembro de 2018, com intervalo de confiança de $95 \%$ Fonte: Elaborado pelos autores com software R (2017)

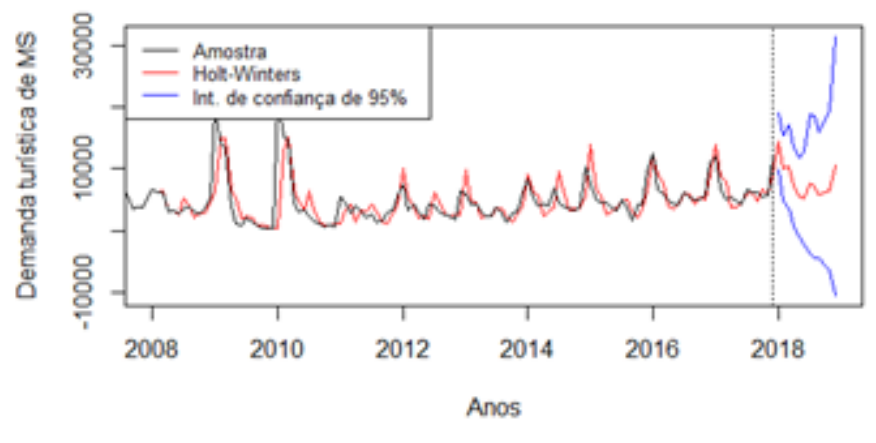

Figura 6 - Previsões de Holt-Winters versão multiplicativa de janeiro até dezembro de 2018, com intervalo de confiança de 95\%

Fonte: Elaborado pelos autores com software R (2017) 


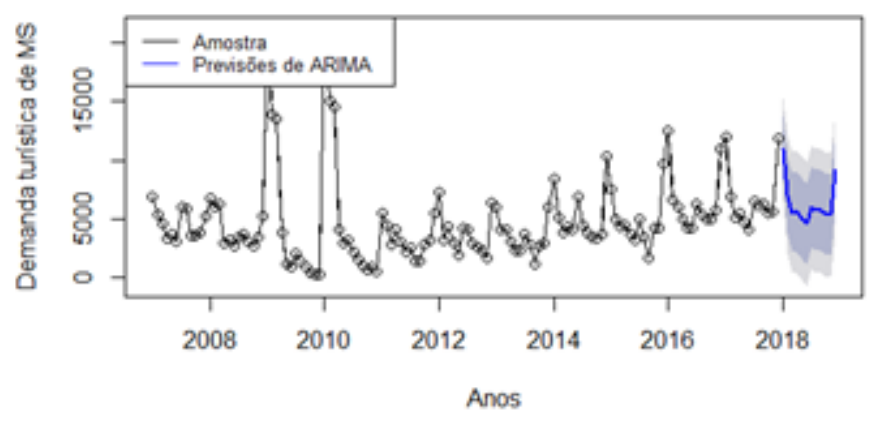

Figura 7 - Previsões de ARIMA de janeiro até dezembro de 2018

Fonte: Elaborado pelos autores com R (2017)

As previsões do modelo ARIMA concernentes aos meses de janeiro a dezembro de 2018 foram comparadas aos dados reais (tabela 3), já disponibilizados pela própria Embratur.

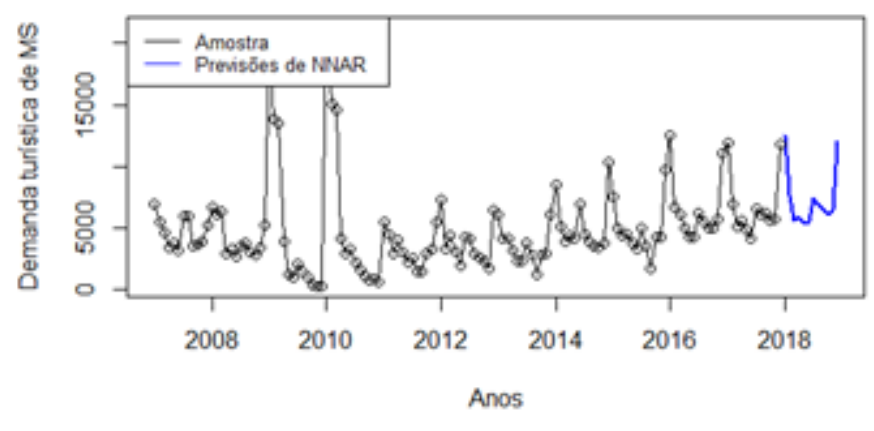

Figura 8 - Previsões de NNAR de janeiro até dezembro de 2018.

Fonte: Elaborado pelos autores com software R (2017)

Os parâmetros de alisamento para as equações de nível, tendência e sazonalidade de ambas as versões de Holt-Winters, o modelo ARIMA selecionado com o menor critério de informação akaike (AIC) e rede neural (NNAR) onde p é o número automaticamente selecionado para série de tempo não sazonal. P é o número de inputs atrasadas e size é o número de nós na camada escondida estes referentes às previsões, demonstrados na tabela 2.

Tabela 2 - Parâmetros das previsões dos modelos

\begin{tabular}{lc}
\hline HW aditiva $(\alpha, \beta, \gamma)$ & HW multiplicativa $(\alpha, \beta, \gamma) \quad$ ARIMA NNAR $(p ; P ; s i z e)$ \\
\hline \hline
\end{tabular}

Fonte: elaborada pelos autores

Os modelos descritos foram utilizados para gerar as previsões no curto, médio e longo prazos das chegadas turísticas. As observações e previsões de Holt-Wiinters versões aditiva, multiplicativa, ARIMA e rede neural em números para os meses de janeiro até dezembro de 2018, podem ser visualizadas na tabela 3. 
Tabela 3 - Comparação dos valores previstos com dados reais

\begin{tabular}{|l|l|l|l|l|l|}
\hline \multicolumn{5}{c}{ Ano-2018 } \\
\hline Meses & Dados reais & HW aditiva & HW multiplicativa & ARIMA & Rede neural (NNAR) \\
\hline Janeiro & 12.573 & 12.607 & 14.368 & 10.927 & 12.569 \\
Fevereiro & 7.550 & 9.383 & 10.119 & 7.081 & 7.622 \\
Março & 7.277 & 9.376 & 10.330 & 5.551 & 5.555 \\
Abril & 5.830 & 7.763 & 7.163 & 5.613 & 5.836 \\
Maio & 5.662 & 6.564 & 5.395 & 5.033 & 5.284 \\
Junho & 5.499 & 6.622 & 5.264 & 4.570 & 5.414 \\
Julho & 7.417 & 7.958 & 7.524 & 6.003 & 7.389 \\
Agosto & 7.707 & 7.716 & 7.092 & 5.707 & 6.873 \\
Setembro & 6.638 & 7.166 & 5.694 & 5.771 & 6.575 \\
Outubro & 6.279 & 7.321 & 6.184 & 5.331 & 6.041 \\
Novembro & 5.767 & 7.309 & 6.345 & 5.412 & 6.446 \\
Dezembro & 13.319 & 10.525 & 10.443 & 9.140 & 11.987 \\
\hline
\end{tabular}

fonte: elaborada pelos autores com base em EMBRATUR (2017)

MAPE, MAD e MSD revelam as diferenças entre os dados reais e valores previstos. Portanto, haverá melhores previsões desempenho se houver quantidades menores desses critérios.

As medidas de erro MAPE, MAD e MSD apresentam a precisão dos modelos em comparação com os dados reais. Uma característica óbvia na tabela 4 é que a rede neural apresentou melhor desempenho do que os modelos concorrentes em todos os horizontes, assim apresentou MAPE inferior a 10\% nos horizontes de previsão de curto, médio e longo prazo. Este percentual é considerado pela classificação de Lewis (1982), como previsão excelente.

Tabela 4 - Medidas de erro

\begin{tabular}{lllll}
\hline Ranking & Modelo e erro por horizonte de previsão (h) & MAPE & MAD & MSD \\
\hline $4^{\circ}$ & HW aditiva $\mathrm{h}=12$ & $16.88 \%$ & 1198.708 & 1455.652 \\
$2^{\circ}$ & HW multiplicativa $\mathrm{h}=12$ & $14.90 \%$ & 1205.444 & 1605.724 \\
$1^{\circ}$ & Rede neural (NNAR) $\mathrm{h}=12$ & $5.88 \%$ & 453.3089 & 713.5987 \\
$3^{\circ}$ & ARIMA $\mathrm{h}=12$ & $15.44 \%$ & 1281.00 & 1644.023 \\
$4^{\circ}$ & HW aditiva $\mathrm{h}=6$ & $20.48 \%$ & 1320.963 & 1504.535 \\
$3^{\circ}$ & HW multiplicativa $\mathrm{h}=6$ & $20.35 \%$ & 1542.061 & 1873.160 \\
$1^{\circ}$ & Rede neural (NNAR) $\mathrm{h}=6$ & $5.64 \%$ & 387.0009 & 738.9662 \\
$2^{\circ}$ & ARIMA $\mathrm{h}=6$ & $12.45 \%$ & 935.4837 & 1096.076 \\
$3^{\circ}$ & HW aditiva $\mathrm{h}=3$ & $17.80 \%$ & 1322.365 & 1609.236 \\
$4^{\circ}$ & HW multiplicativa $\mathrm{h}=3$ & $30.09 \%$ & 2472.669 & 2526.388 \\
$1^{\circ}$ & Rede neural (NNAR) $\mathrm{h}=3$ & $8.29 \%$ & 605.5967 & 1016.395 \\
$2^{\circ}$ & ARIMA $\mathrm{h}=3$ & $14.33 \%$ & 1279.77 & 1402.861 \\
\hline \multicolumn{4}{l}{} \\
\hline
\end{tabular}

h é o horizonte de previsão curto prazo três meses, médio prazo seis meses e longo prazo 12 meses Fonte: elaborada pelos autores

No curto, médio e longo prazo a rede neural superou os modelos ARIMA, HW ambas versões, assim foi o modelo mais preciso em comparação aos demais modelos aplicados. Em todos os critérios MAPE, MAD e MSD, a rede neural gerou os melhores indicadores de qualidade da robustez do modelo de previsão. Este 
resultado é relevante, pois entre os modelos comparados a rede neural é o melhor modelo para prever a demanda turística do estado de Mato Grosso do Sul (MS).

O segundo melhor desempenho individual no curto e médio prazo foi o modelo ARIMA (14,33\%), (12.45\%), mas de longo prazo HW (14.90\%) versão multiplicativa gerou a segunda previsão mais precisa com base nos resultados dos critérios MAPE, MAD e MSD como pode ser observada na tabela 4.

De acordo com as diretrizes de Lewis (1982), os resultados de MAPE entre 10\% e 20\% são interpretados como previsão precisa, diante disso, os modelos que geraram o segundo desempenho mais preciso apresentaram boas previsões.

\section{CONSIDERAÇÕES FINAIS}

Quanto aos resultados obtidos na pesquisa, mostrou-se que a rede neural (NNAR) fornece previsões mais precisas da procura do turismo do MS, do que ARIMA e Holt-Winters (versões aditiva e multiplicativa). Esses achados estão consistentes com o que foi constatado por Szoplik (2015), que os modelos de previsão de rede neural superam os métodos de série de tempo em termos de precisão. Os resultados também estão consistentes com o que foi constatado por (CHO, 2003).

Além disso, o MAPE de 5,64\% deste estudo alcançou resultados próximos do MAPE (3,44\%) obtidos pela previsão complexa da rede neural perceptron multicamada (MLP) da demanda turística na pesquisa de (PALMER; JOSÉ MONTAÑO; SESÉ, 2006). Os resultados indicam a viabilidade de utilização da rede neural para prever a demanda turística de MS.

A rede neural aplicada nesta pesquisa serve para auxiliar no processo de tomada de decisão do planejamento da atividade turística, uma vez que ter uma previsão precisa possibilita ao gestor melhoria na utilização dos recursos financeiros e humanos de um empreendimento turístico. Os modelos podem apoiar as decisões dos planejadores turísticos da Fundação de Turismo de Mato Grosso do Sul e a Secretaria Municipal de Turismo de Bonito com relação à redução das incertezas quanto à quantidade de chegadas turísticas (fluxo turístico) no estado.

A pesquisa limitou-se em uma única série temporal e, além disso, a série não foi submetida aos testes de raízes unitárias Dickey-Fuller ou Dickey-Fuller Aumentado. Para superar essa limitação sugere-se a estudos futuros executar a previsão e utilizar um amplo número de séries temporais (no mínimo dez) para constatar o nível de generalização dos resultados verificados, isso também é recomendável por conta dos resultados de pesquisas anteriores (GOH; LAW, 2002; SONG; LI, 2008; HASSANI et al., 2017).

\section{ReFERENCIAS}

ARCGIS. ESRI California: ESRI10.1. Redlands(Software), 2012.

BARBOSA, M. A. C.; ZAMBONI, R. A. Formação de um "cluster" em torno do turismo de natureza sustentável em Bonito-MS. Disponível em: . Acesso em: 14 ago. 2017.

BONETT, D. G.; SEIER, E. Statistical Inference for a Ratio of Dispersions Using Paired Samples. Journal of Educational and Behavioral Statistics, v. 28, n. 1, p. 21-30, 2003.

BRANDON, C.; JARRETT, J. E.; KHUMAWALA, S. B. A Comparative Study of the Forecasting Accuracy of Holt\# Winters and Economic Indicator Models of Earnings Per Share For Financial Decision Making. Managerial Finance, v. 13, n. 2, p. 10-15, 1987.

BROCKWELL, P. J.; DAVIS, R. A. Time series: theory and methods. [s.l.] Springer-Verlag, 1991.

BUNDCHEN, C. Previsão de demanda turística e a acurácia das previsões frente à realização de megaeventos. 2016. Dissertação (Mestrado em Engenhari de Produção ), Programa de Pós graduação em Engenharia de Produção - Universidade Federal do Rio Grande do Sul, Porto Alegre. 
Bruno Matos Porto, et al. Previsão dos modelos univariados e rede neural da demanda turística do e...

BUNDCHEN, C.; WERNER, L. Comparação da Acurácia de Previsões de Demanda Turística em Sedes Olímpicas. Turismo em Análise, v. 27, n. 1, p. 85-107, 2016.

CASANOVA, S. et al. Previsão da Demanda Turística da Cidade de Foz do Iguaçu: Uma Aplicação com os Modelos ARIMA. Revista Turismo Visão e Ação, v. 14, n. 3, p. 366-385, 2012.

CHATFIELD, C. The Holt-Winters Forecasting Procedure. Journal of the Royal Statistical Society, v. 27, n. 3, p. 264-279, 1978.

CHATFIELD, C. Apples, oranges and mean square error. International Journal of Forecasting, v. 4, n. 4, p. 515$518,1988$.

CHATFIELD, C.; YAR, M. Holt-Winters forecasting\#: some practical issues. Journal of the Royal Statistical Society. Series D (The Statistician), v. 37, n. 2, p. 129-140, 1988.

$\mathrm{CHO}, \mathrm{V}$. A comparison of three different approaches to tourist arrival forecasting. Tourism Management, v. 24, p. 323-330, 2003.

CHU, F.-L. Forecasting tourism demand in asian-pacific countries. Annals of Tourism Research, v. 25, n. 3, p. 597615, 1998.

CLAVERIA, O.; TORRA, S. Forecasting tourism demand to Catalonia: Neural networks vs. time series models. Economic Modelling, v. 36, p. 220-228, 2014.

EMBRATUR. Turismo já ajudou países a superar crises e pode fazer o mesmo no Brasil. Disponível em: . Acesso em: 22 jun. 2017a.

EMBRATUR. O que é. Disponível em: . Acesso em: 29 jun. 2017b.

EMBRATUR. Para que. Disponível em: . Acesso em: 29 jun. 2017c.

EMBRATUR. Termos e condiçôes de uso. Disponível em: . Acesso em: 29 jun. 2017d.

EMBRATUR. Acesse o extrator de dados. Disponível em: . Acesso em: 29 jun. 2017e.

FLORES, B. E. A pragmatic view of accuracy measurement in forecasting. Omega, v. 14, n. 2, p. 93-98, 1986.

GOH, C.; LAW, R. Modeling and forecasting tourism demand for arrivals with stochastic nonstationary seasonality and intervention. Tourism Management, v. 23, n. 5, p. 499-510, 2002.

GOH, C.; LAW, R.; MOK, H. M. K. Analyzing and forecasting tourism demand: A rough sets approach. Journal of Travel Research, v. 46, n. 3, p. 327-338, 2008.

GUNTER, U.; ÖNDER, I. Forecasting international city tourism demand for Paris: Accuracy of uni- and multivariate models employing monthly data. Tourism Management, v. 46, p. 123-135, 2015.

HASSANI, H. et al. Forecasting accuracy evaluation of tourist arrivals. Annals of Tourism Research, v. 63, p. 112127, 2017.

HYNDMAN, R. et al. Forecasting Functions for Time Series and Linear Models Package 'forecast'CRAN, 2018. Disponível em:

HYNDMAN, R. J.; ATHANASOPOULOS, G. 8.7 ARIMA modelling in R. In: Forecasting: principles and practice. Melbourne, Australia: OTexts, 2018a.

HYNDMAN, R. J.; ATHANASOPOULOS, G. 11.3 Neural network models. In: Forecasting: principles and practice. Melbourne, Australia: OTexts, 2018b.

HYNDMAN, R. J.; ATHANASOPOULOS, G. 1.6 The basic steps in a forecasting task. In: Forecasting: principles and practice. Melbourne, Australia: OTexts, 2018c.

HYNDMAN, R. J.; KHANDAKAR, Y. Automatic time series forecasting: The forecast package for R. Journal Of Statistical Software, v. 27, n. 3, p. 2-22, 2008.

IBGE. Estados. Disponível em: . Acesso em: 23 nov. 2017.

KAZMIER, L. J. Estatística aplicada à economia e administração. São Paulo: McGraw-Hill do Brasil, 1982.

KIM, J. H. et al. Beyond point forecasting: Evaluation of alternative prediction intervals for tourist arrivals. International Journal of Forecasting, v. 27, n. 3, p. 887-901, 2011. 
LAW, R. Back-propagation learning in improving the accuracy of neural network-based tourism demand forecasting. Tourism Management, v. 21, n. 4, p. 331-340, 2000.

LEE, R.; QIAN, M.; SHAO, Y. On Rotational Robustness of Shapiro-Wilk Type Tests for Multivariate Normality. Open Journal of Statistics, v. 4, n. December, p. 964-969, 2014.

LEWIS, C. D. Industrial and business forecasting methods: A practical guide to exponential smoothing and curve fitting. Butterworth-Heinemann, 1982.

MAKRIDAKIS, S. A Survey of Time Series. International Statistical Review / Revue Internationale de Statistique, v. 44, n. 1, p. 29, 1976.

MAKRIDAKIS, S. Accuracy concerns measures: theoretical and practical concerns. International journal of forecasting, v. 9, p. 527-529, 1993.

MATO GROSSO DO SUL. Dossiê de candidatura à Rede Global de Geoparks Nacionais sob auspício da Organização das Nações Unidas para Educação, Ciências e Cultura. Disponível em: . Acesso em: 14 ago. 2017, 2010.

MATO GROSSO DO SUL. Fundação de Turismo de MS-FUNDTUR: Indicadores Turísticos de MS. Disponível em: . Acesso em: 14 ago. 2017, 2017.

MIN, J. C. H. Forecasting Japanese tourism demand in Taiwan using an intervention analysis. International Journal of Culture, Tourism and Hospitality Research, v. 2, n. 3, p. 197-216, 2008.

MINITAB Inc. Statistical Software Data Analysis Software. Version 18.1.0

NEWBOLD, P.; GRANGER, C. W. J. Experience with Forecasting Univariate Time Series and the Combination of Forecasts. Wiley for the Royal Statistical Society, v. 137, n. 2, p. 131-165, 1974.

PALMER, A.; JOSÉ MONTAÑO, J.; SESÉ, A. Designing an artificial neural network for forecasting tourism time series. Tourism Management, v. 27, n. 5, p. 781-790, 2006.

ROTTA, M. A.; LUNA, H. S. E; WEIS, W. A. Ecoturismo no Pantanal. Disponível em: . Acesso em: 14 ago. 2017.

SERRA, C. M. V.; TAVARES, H. R.; SANTOS, J. C. C. Aplicação de séries temporais na análise de demanda turística no estado do Pará usando os modelos de Holt-Winters. In: Encontro Nacional de Engenharia de Produção ENEGEP, XXV, 2005. Anais... Porto Alegre: 2005. Disponível em:

SILVER, M. Estatística para administração. São Paulo: Atlas, 2000.

SONG, H.; LI, G. Tourism demand modelling and forecasting-A review of recent research. Tourism Management, v. 29, n. 2, p. 203-220, 2008.

STEVENSON, W. J. Estatística aplicada à administração. São Paulo: Harper \& Row do Brasil, 1981.

SZOPLIK, Jolanta. Forecasting of natural gas consumption with artificial neural networks. Energy, v. 85, p. 208-220, 2015.

THOMPSON, P. A. An MSE statistic for comparing forecast accuracy across series. International Journal of Forecasting, v. 6, n. 2, p. 219-227, 1990.

CC BY 